\title{
Kinked coronary saphenous vein graft after aortic dissection surgery: An unusual differential diagnosis of cardiac tamponade
}

Kaushalendra Rathore, F.R.A.C.S. ${ }^{(1)}$

Mark Teh, M.D. ${ }^{(2)}$

Mark Newman, F.R.A.C.S. ${ }^{(1)}$

From ${ }^{(1)}$ Department of Cardiothoracic Surgery, Sir Charles Gairdner Hospital, Hospital Avenue, Nedlands WA 6009 Australia.

(2) Department of Radiology, Perth Radiology Clinic, Perth WA 6000 Australia. Address correspondence to K.R. (e-mail: kaushalendra.rathore@health.wa.gov.au)

Received 7 February 2021 ; revised 5 July 2021 ; accepted 9 August 2021

doi:10.46475/aseanjr.v22i2.118

\section{Abstract}

A 73-year-old female patient presented with cardiac tamponade following Stanford type A aortic dissection required immediate pericardiocentesis and followed by root replacement with coronary artery bypass grafting. The patient's postoperative course was eventful and coronary graft kinking contributed to myocardial ischemia, but the presentation was confused with cardiac tamponade.

Keywords: Angina, Shock, Coronary artery disease. 


\section{Introduction}

Long-term outcomes of aortic root replacement using coronary ostial buttons have been stated with good results and there are no concerns emerging in the follow-up process regarding coronary button anastomosis [1]. However, post-operative ischemic issues related to the coronary buttons, especially in the high-risk patients of type A aortic dissection involving aortic root have been well-reported, and still pose challenging clinical circumstances.

\section{Case summary}

A 73-year-old female was presented with cardiogenic shock which was followed by cardiac arrest in the emergency department, and it was managed with a brief period of cardiopulmonary resuscitation. The patient's cardiac output returned and immediate bedside Transthoracic Echocardiogram (ECHO) revealed large haemorrhagic pericardial collection with the features of cardiac tamponade which was drained percutaneously using an $8.3 \mathrm{~F}$ catheter under guidance. Cardiac gated contrast Computed Tomography (CT scan) displayed Stanford type A aortic dissection extending from the aortic root proximally and a ruptured Penetrating Aortic Ulcer (PAU) posteriorly near the coronary ostia (Figure 1A, 1B and 2A). Intra-operative Trans Oesophageal Echocardiogram (TEE) showed a tri-leaflet aortic valve with severe regurgitation. Aortic root replacement with replacement of the ascending aorta including hemiarch using deep circulatory arrest was performed (23 Magna ease, Edward life science, USA, and $26 \mathrm{~mm}$ Gelweave Dacron tube graft, Terumo, USA) and coronary ostia were implanted as buttons over the Gelweave graft. Dissection flap was extending well beyond RCA button, and it led to severe bleeding after coming off cardiopulmonary bypass. Eventually, reverse Saphenous Vein (SVG) graft over mid RCA in end to side manner and the proximal end was grafted on the Gelweave graft, while proximal native RCA was disconnected and ligated. 

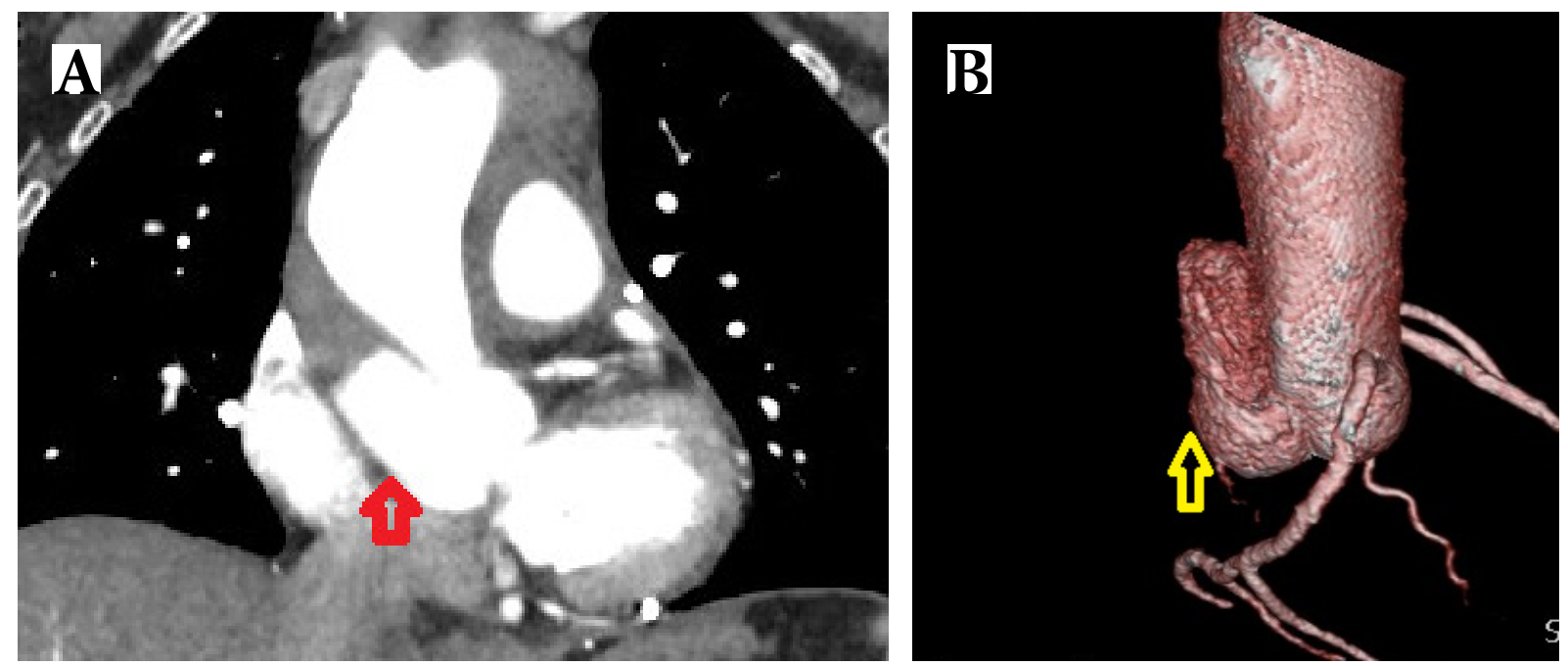

Figure 1. (A) Contrast CT scan: Arrow showing penetrating aortic ulcer (PAU) and dissection extending to the root. (B) 3-Dimensional volume rendered view of contrast CT scan: arrow showing penetrating aortic ulcer (PAU).
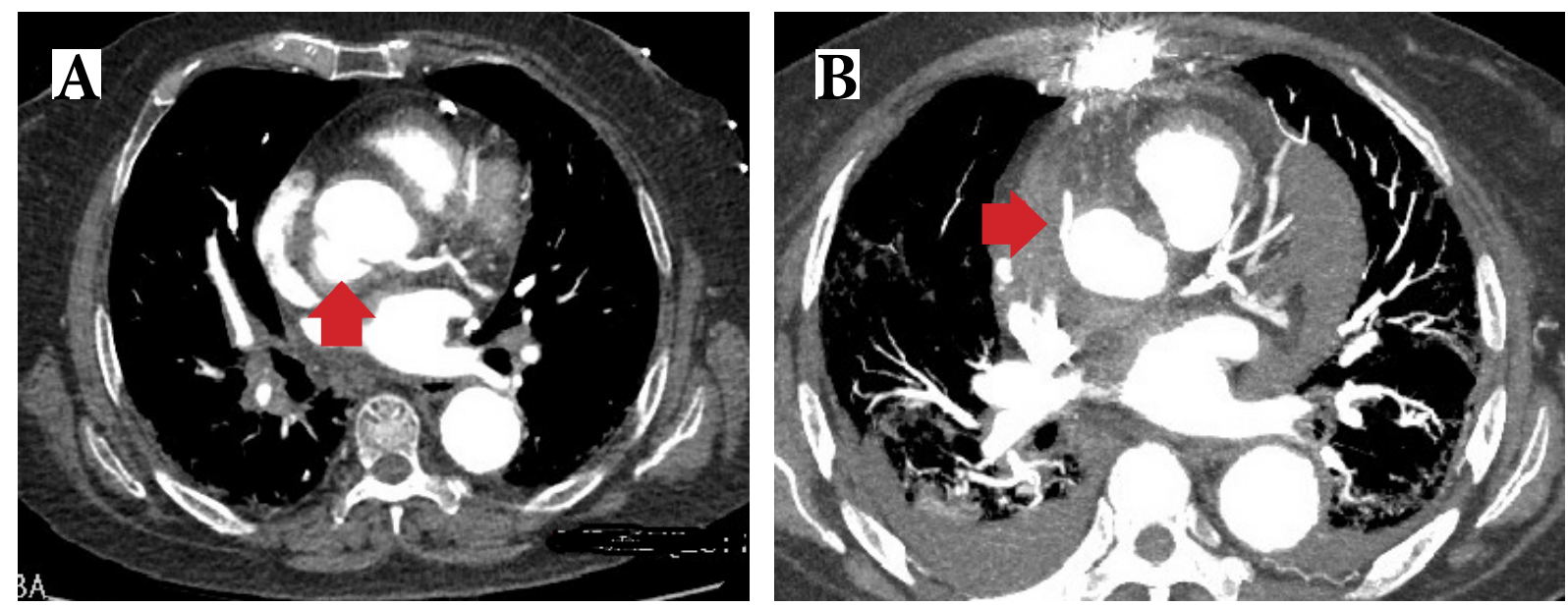

Figure 2. (A) Contrast coronary angiogram (axial view) arrow showing aortic dissection in proximity with the root and left coronary ostia. (B) Post-operative contrast coronary angiogram (axial view) arrow showing saphenous vein graft surrounded by the pericardial fluid collection. 


\section{Imaging findings}

Post-operative follow-up contrast CT chest showed good patency of the vein graft with moderate pericardial collection, which was assumed to be reactionary fluid from Gelweave graft and managed conservatively (Figure 2B). The patient developed hypotension and bradycardia ten days after the operation and underwent urgent surgical pericardial drainage with a working diagnosis of cardiac tamponade, although intra-operative TEE showed no features of cardiac tamponade. The patient was discharged to rehabilitate but represented after three weeks with acute onset angina at rest with very high Troponin I level (15000 nanogram/litre) and diagnosed with inferior ST elevation Myocardial Infarction (STEMI). Trans-Thoracic Echocardiogram (TTE) showed severe Right Ventricular (RV) dysfunction with inferior wall hypokinesia, and the patient was referred for urgent coronary angiogram (CAG). CAG showed kinking of the SVG ostia (Figure 3A), which was opened by deploying two Promus PREMIER Drug Eluting Stents (Boston scientific, USA) (Figure 3B). The follow-up contrast CT aortogram confirmed patency of the stent in the proximal SVG with patent distal vein graft (Figure 4). The patient recovered after the coronary intervention and TTE prior to the discharge revealed improving RV systolic functions. 

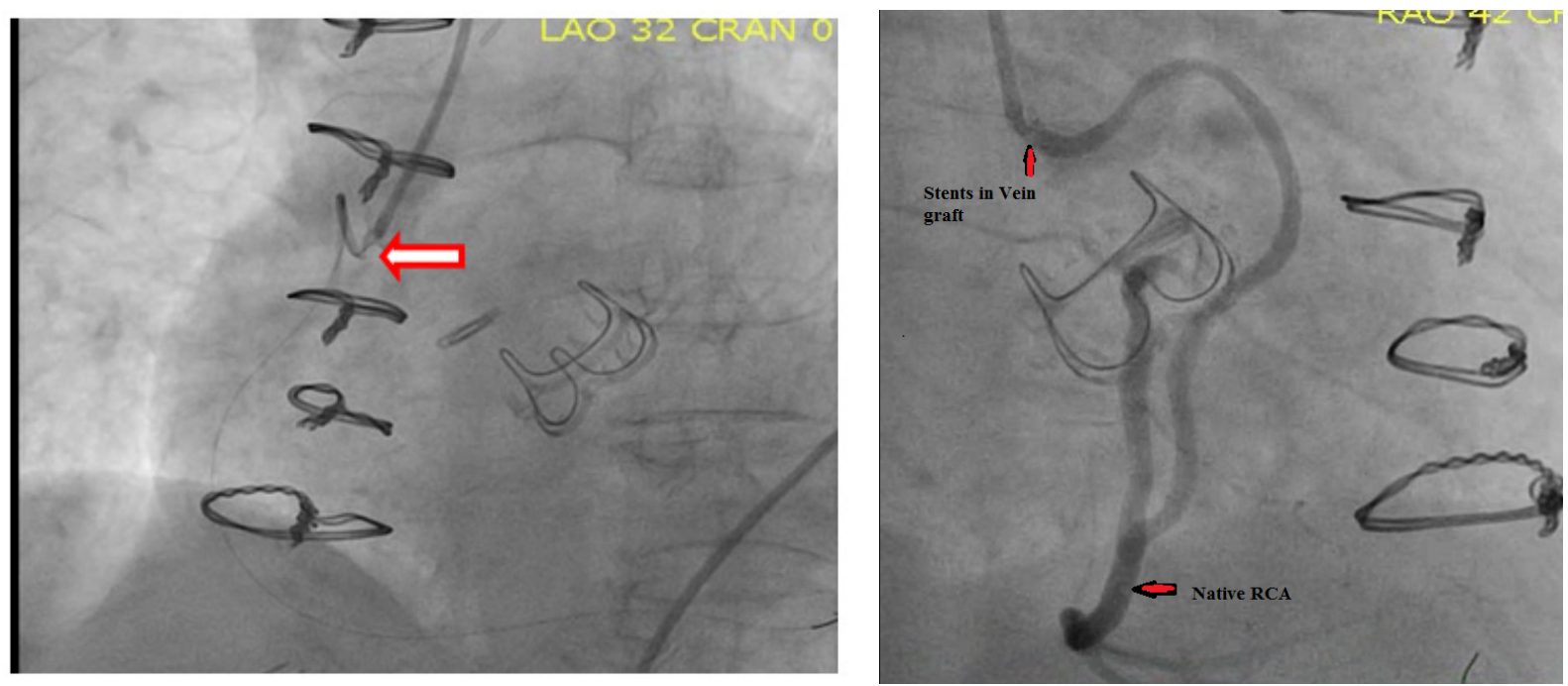

Figure 3. (A) Coronary angiogram: arrow showing SVG ostia stenosis with guidewire in-situ in the SVG lumen. (B) Coronary Angiogram: arrow showing SVG after the stent deployment.

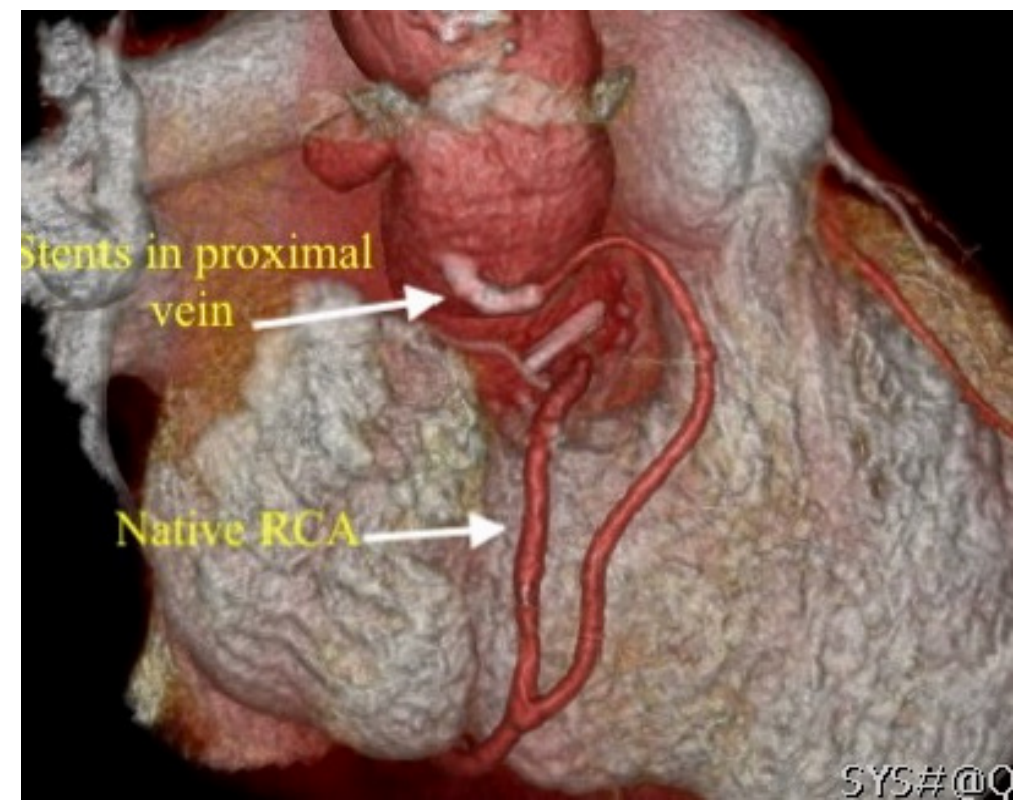

Figure 4. Post-operative contrast CT scan: volume rendered image showing coronary stents in the SVG. 


\section{Discussion}

Even with continuous improvement in the operative techniques, all-inclusive surgical morbidity, and mortality of acute Type A Aortic Dissection (TAAD) remained high (17\%-26\%) and the overall prognosis of a TAAD patient is determined by the patient's comorbidities and dissection-related complications, like unstable patients with circulatory malperfusion and the extent of surgical repair [1]. Malperfusion secondary to the TAAD is well known (20-35\%) and it can involve coronary arteries, arch vessels, visceral arteries, or distal femoral arteries [2]. Acute myocardial infarction after TAAD surgery is reported between 5.6-5.9\% in a systemic review and continues to be a major life-threatening situation [2].

Patients with TAAD can have retrograde dissection flap extension into the coronary ostia or further distally into the main coronary artery and its branches. Neri et al. had differentiated three main types of coronary lesions, coronary ostial dissection, and dissection with a coronary false channel and circumferential detachment with an inner cylinder intussusception [3]. Although various techniques have been described to repair dissected coronary artery during an index aortic repair, because of the good results and simplicity, the most commonly used technique is a coronary bypass graft while ligating native coronary artery [4].

Iatrogenic kinking or stenosis of the vein graft is also well known and most often this issue arises between two weeks to six months after the surgery, as it happened in our case. Various mechanisms have been reported in isolated case reports, namely, mechanical obstruction (compression from the haematoma surrounding the heart, fluid and blood leading to tamponade and bioglue) and dynamic obstruction, for example, low or high positioning of the SVG on the tube graft, ostial obstruction due to the excessive or short length of the graft [5]. Presentation may vary from immediate post-operative cardiogenic shock, rhythm changes and cardiac arrest to late presentation with STEMI and heart failure. Management depends upon the stage of the presentation and mechanism responsible for these changes. Surgery can be an option for the mechanical obstruction and where the patient needs a release of the cardiac tamponade, while 
the majority of the cases are treated with Per Cutaneous Interventions (PCI) using drug eluting stents [5]. Some benefits of PCI are fast revascularization and limiting ischemic myocardial damage, less invasion and good outcomes.

Our patient had graft flow issues on day ten which leads to a low cardiac output with RV dysfunction in the post-operative period, but it was confused with cardiac tamponade and ended in unfruitful surgical re-exploration and delay in the correct diagnosis. These patients should be further triaged using TTE, blood biochemistry markers (Troponins) and if doubt persists then urgent CT coronary angiogram should be performed as delay in the treatment can be detrimental.

\section{Conclusion}

Our case again re-enforces the fact that the acute myocardial infarction after TAAD repair should be diagnosed promptly and managed effectively using either options in surgery or per-cutaneous interventions to avoid possible ischemic complications. 


\section{References}

1. Mookhoek A, Korteland NM, Arabkhani B, Di Centa I, Lansac E, Bekkers JA, et al. Bentall procedure: a systematic review and meta-analysis. Ann Thorac Surg 2016;101:1684-9. doi: 10.1016/j.athoracsur.2015.10.090.

2. Helder MRK, Schaff HV, Day CN, Pochettino A, Bagameri G, Greason KL, et al. Regional and temporal trends in the outcomes of repairs for acute type a aortic dissections. Ann Thorac Surg 2020;109:26-35. doi: 10.1016/j.athorac sur.2019.06.058.

3. Neri E, Toscano T, Papalia U, Frati G, Massetti M, Capannini G, et al. Proximal aortic dissection with coronary malperfusion: presentation, management, and outcome. J Thorac Cardiovasc Surg 2001;121:552-60. doi: 10.1067/ mtc.2001.112534.

4. Tribble C. To know acute aortic dissection is to know all of cardiovascular surgery. Semin Thoracic Surg 2017; 29:186-7. doi: 10.1053/j.semtcvs.2017.01. 014.

5. Adamson C, Rocchiccioli P, Brogan R, Berry C, Ford TJ. Post-operative myocardial infarction following aortic root surgery with coronary reimplantation: a case series treated with percutaneous coronary intervention. Eur Heart J Case Rep 2019; 3:1-6. doi: 10.1093/ehjcr/ytz181. 